European

Thyroid Journal
Eur Thyroid J 2019;8:1-6

DOI: $10.1159 / 000494430$
Received: July 27, 2018

Accepted after revision: October 9, 2018

Published online: November 22, 2018

\title{
Evaluation of Diagnostic Utility of Immunohistochemistry Markers of TROP-2 and HBME-1 in the Diagnosis of Thyroid Carcinoma
}

\author{
Nooshin Zargari Maral Mokhtari \\ Pathology Department, Shiraz University of Medical Sciences, Shiraz, Iran
}

\section{Keywords}

Thyroid - Papillary thyroid carcinoma - Follicular carcinoma . Follicular adenoma $\cdot$ Multinodular goiter $\cdot$ TROP-2 $\cdot$ HBME-1

\begin{abstract}
Objective: The diagnosis of thyroid tumors is based on histologic features, but the distinction between benign and malignant thyroid lesions is somehow difficult, especially in lesions with a follicular pattern. Thus, ancillary studies, such as immunohistochemistry (IHC), may be helpful. In this study, we aimed to evaluate the diagnostic value of $2 \mathrm{IHC}$ markers, TROP-2 and HBME-1, in the diagnosis of thyroid cancers. Study Design: A total of 102 cases of thyroid lesions, including papillary thyroid carcinoma (classic variant) (29 cases), follicular variant of papillary thyroid carcinoma (21 cases), multinodular goiter ( 22 cases), follicular adenoma ( 24 cases), and follicular carcinoma (6 cases) were selected. The hematoxylin-eosin slides were reviewed for confirmation of diagnosis, and IHC examination for TROP-2 and HBME- 1 was performed. Result: HBME-1 had $84 \%$ sensitivity and $98 \%$ specificity with a positive predictive value (PPV) of $98 \%$ and a negative predictive value (NPV) of $83 \%$. TROP-2 had $93 \%$ sensitivity and $74 \%$ specificity with a PPV of $81 \%$ and an NPV
\end{abstract}

of $90 \%$ in malignant thyroid lesions. Conclusion: IHC panel consisting of TROP-2 and HBME-1 can be used in equivocal follicular patterned lesions for the diagnosis of thyroid carcinomas.

(c) 2018 European Thyroid Association Published by S. Karger AG, Basel

\section{Introduction}

Thyroid lesions with a follicular pattern, which is defined as the cells arranged around a central lumen containing colloid material, consist of a large group of thyroid neoplasms, including follicular adenoma (FA), follicular carcinoma (FC), and follicular variant of papillary thyroid carcinoma (FVPTC). The standard diagnosis of thyroid tumors is based on hematoxylin-eosin (HE)stained slides, but sometimes the diagnosis of thyroid lesions, especially follicular patterned thyroid neoplasms, is difficult because of overlapping histomorphology. For example, nuclear features of PTC may be focal and subjective, or interpretation of capsular or vascular invasion may pose diagnostic dilemma, so utility of immunohistochemical (IHC) markers may be helpful in differentiating between benign and malignant thyroid lesions [1-5]. Pre- 
vious studies evaluated the utility of several markers, such as CK19, galectin 3, Hector Battifora mesothelial-1 (HBME-1), CD44, CD57, Cyclin D1, P27, and trophoblastic cell surface antigen-2 (TROP-2) [2-14]. HBME-1 is a membrane antigen found in the mesothelial cells and in lung, breast, and pancreas adenocarcinomas. TROP-2, also known as tumor-associated calcium signal transducer 2 (TAC-STD2) $[6,8,11]$, is a $35-\mathrm{kDa}, 323$ amino acid, type 1 transmembranous glycoprotein. It is identified in choriocarcinoma and trophoblastic cells. Its overexpression is seen in the majority of human carcinomas, including colorectal, gastric, and pancreatic adenocarcinoma, squamous cell carcinoma of the oral cavity, non-small cell carcinoma of the lung, and endometrial and ovarian cancers, and rarely in normal tissue. TROP-2 overexpression in carcinoma is related to poor prognosis and aggressive behavior $[6,8,11]$, but no single marker is sensitive nor specific for differentiation between benign and malignant thyroid neoplasm. Therefore, in this study, we evaluated the diagnostic utility of a panel consisting of HBME- 1 and TROP-2 for differentiation between benign and malignant thyroid lesions.

\section{Material and Method}

During 2012 and 2017, the archive of the Surgical Pathology Department of Shahid Rajaee and Faghihi Hospitals, affiliated to Shiraz University of Medical Sciences, Shiraz, Iran, was searched for cases of benign and malignant thyroid lesions, diagnosed on total or subtotal thyroidectomy or lobectomy specimens. The ethics committee of Shiraz University of Medical Sciences approved the study. The HE slides were reviewed by a pathologist for confirmation of the diagnosis. If the diagnosis was not definite, a second pathologist was consulted for definite diagnosis.

The lesion was regarded as PTC if showing nuclear features of PTC, i.e., (1) nuclear enlargement/overlapping/crowding or elongation, (2) nuclear membrane irregularities, grooves, or pseudoinclusions, or (3) glassy nuclei/cleared chromatin with margination; as FA if showing small or large follicles with central colloid in a background of euthyroid tissue without vascular or capsular invasion; as FC if showing vascular or capsular invasion; as FVPTC if composed of follicles with nuclear features of PTC; as noninvasive follicular neoplasm with PTC nuclear features (NIFTP) if the tumor was encapsulated with predominantly follicular architecture and PTC nuclear features, no invasion (capsular or vascular), solid component $<30 \%$, mitotic count of $<3 / 10$ high power field, and no necrosis; as multinodular goiter (MNG) if showing huge follicles lined by flattened epithelium; as Hurthle cell adenoma (HCA) when the lesion was composed of follicular cells with oncocytic change; and as Hurthle cell carcinoma (HCC) when capsular and/ or vascular invasion was seen in the oncocytic lesion. For IHC study, the representative paraffin blocks were selected, and the IHC procedure was done based on the protocol. Table 1 shows the antibodies used in the study.
Table 1. The result of IHC markers in different thyroid lesions

\begin{tabular}{lrll}
\hline Thyroid lesions & $n$ & $\begin{array}{l}\text { HBME-1 } \\
\text { positivity, } \\
n / \text { total } n(\%)\end{array}$ & $\begin{array}{l}\text { TROP-2 } \\
\text { positivity, } \\
n / \text { total } n(\%)\end{array}$ \\
\hline MNG & 22 & $0 / 22(0)$ & $3 / 33(13.6)$ \\
FA and HCA & 24 & $1 / 24(4.2)$ & $9 / 24(37.5)$ \\
FC and HCC & 6 & $3 / 6(50)$ & $5 / 6(83.3)$ \\
PTC & 29 & $27 / 29(93.1)$ & $27 / 29(93.1)$ \\
FVPTC & 19 & $15 / 19(79)$ & $18 / 19(95)$ \\
NIFPT & 2 & $2 / 2(100)$ & $2 / 2(100)$ \\
\hline
\end{tabular}

IHC, immunohistochemistry; MNG, multinodular goiter; FA, follicular adenoma; HCA, Hurthle cell adenoma; FC, follicular carcinoma; HCC, Hurthle cell carcinoma; PTC, papillary thyroid carcinoma; FVPTC, follicular variant of papillary thyroid carcinoma; NIFPT, noninvasive follicular neoplasm with PTC nuclear features.

Immunostained slides were evaluated by a pathologist. For HBME-1 (clone HBME-1, Master Diagnóstica, Spain), cytoplasmic staining with membranous accentuation in $>10 \%$ of cells was regarded as positive, and for TROP-2 (clone B-9, Medaysis, Alameda, CA, USA), cases with no staining or staining in $<5 \%$ of cells were recorded as negative. Only membranous staining of $>5 \%$ of the cells was considered positive: $1+(5-25 \%), 2+(26-50 \%), 3+$ $(51-75 \%)$, and $4+(>75 \%)$.

Data analysis was done using SPSS 22. The sensitivity, specificity, positive predictive value (PPV), and negative predictive value (NPV) of each marker and combination of both markers (HBME1 and TROP-2) were evaluated in comparison to the gold standard represented by the morphologic diagnosis.

\section{Results}

A total of 102 cases of thyroid lesions were retrospectively included in this study and categorized into 5 groups by histologic examination based on morphologic pattern and differentiation. Thus, we had 22 cases of MNG, 24 cases of FA and HCA, 6 cases of FC and HCC, 29 cases of PTC, and 21 cases of FVPTC ( 2 of them regarded as NIFTP). Each lesion was stained with HBME-1 and TROP-2. Table 1 summarizes the results of IHC evaluation in various thyroid lesions.

None of the 22 cases of MNG was positive for HBME1 , and only 3 cases $(3 / 22,13.6 \%)$ showed reactivity with TROP-2. Two cases $(2 / 22,9.1 \%)$ showed $1+$ staining, and 1 case showed $4+$ staining $(1 / 22,4.5 \%)$.

Of the 24 cases of FA and HCA, 1 (4.2\%) was positive for HBME-1 and 9 (37.5\%) were positive for TROP-2. Three $(12.5 \%)$ cases showed $1+$ immunostaining, 1 case
Eur Thyroid J 2019;8:1-6 DOI: $10.1159 / 000494430$
Zargari/Mokhtari 

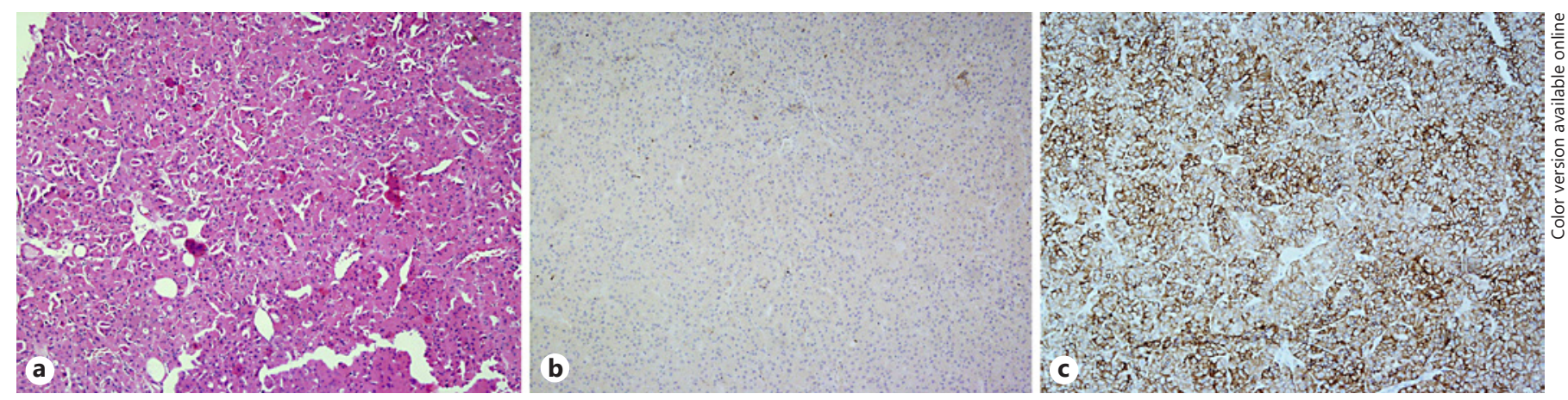

Fig. 1. a Hurthle cell adenoma, HE, $\times 400$. b Negative for HBME-1. c Positivity for TROP-2 in Hurthle cells.
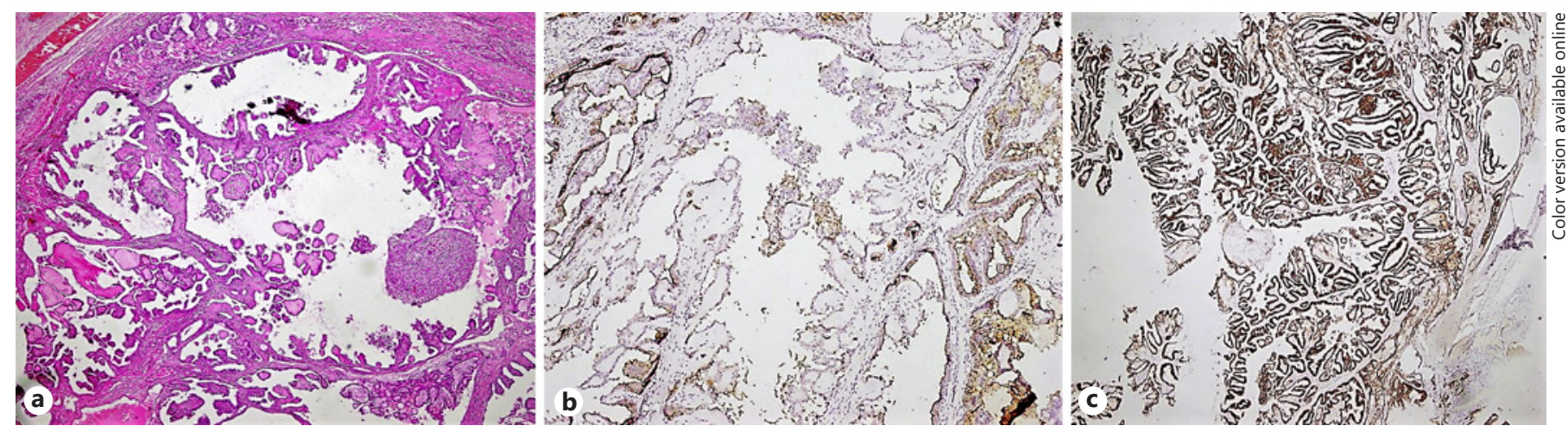

Fig. 2. a Papillary thyroid carcinoma, HE, $\times 250$. b Positive for HBME-1. c TROP-2 immunostaining.
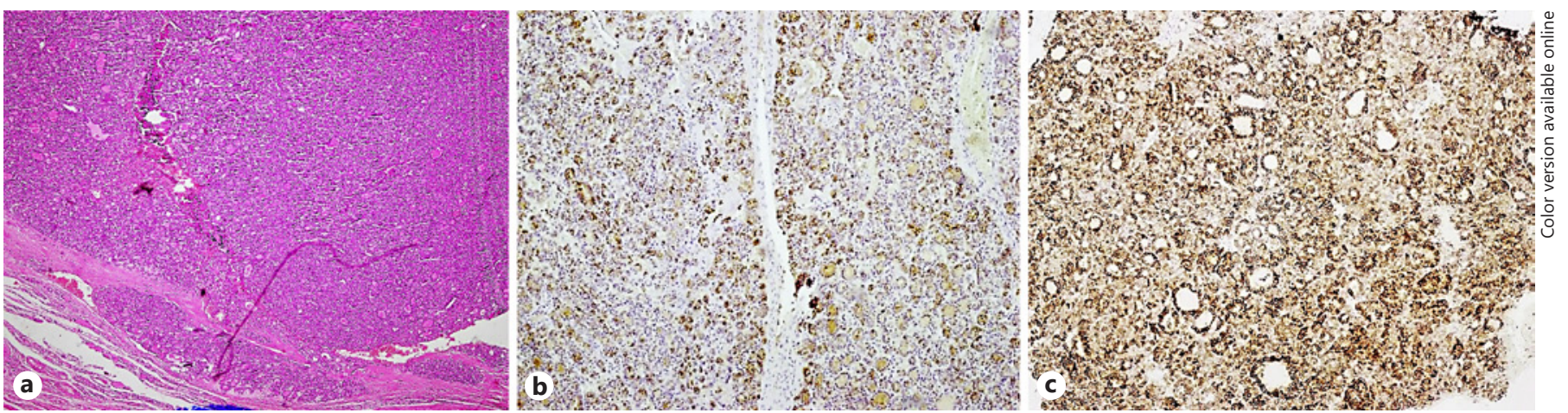

Fig. 3. a Follicular carcinoma with capsular invasion, HE, $\times 250$. b Positive for HBME-1. c TROP-2 positivity.

(4.2\%) showed 2+ immunostaining, $1(4.2 \%)$ showed 3+ immunostaining, and 4 cases (16.7\%) showed $4+$ immunostaining. In this group, all HCAs were positive for TROP-2 (Fig. 1).

Of the 6 cases of FC and HCC (5 FC and 1 HCC), 3 (50\%) cases of FC were positive for HBME-1, but the HCC case was negative for HBME-1. Five (83.3\%) of them (4 FC and 1 HCC) were positive for TROP-2. One case (16.7\%) showed 1+ immunostaining, 1 (16.7\%) showed $2+$ immunostaining, $1(16.7 \%)$ showed $3+$ immunostaining, and 2 cases (1 FC and 1 HCC) showed 4+ immunostaining (33.3\%) (Fig. 2).

Twenty-seven out of 29 cases (93.1\%) of conventional variant of PTC were positive for HBME-1; also, 27 cases 

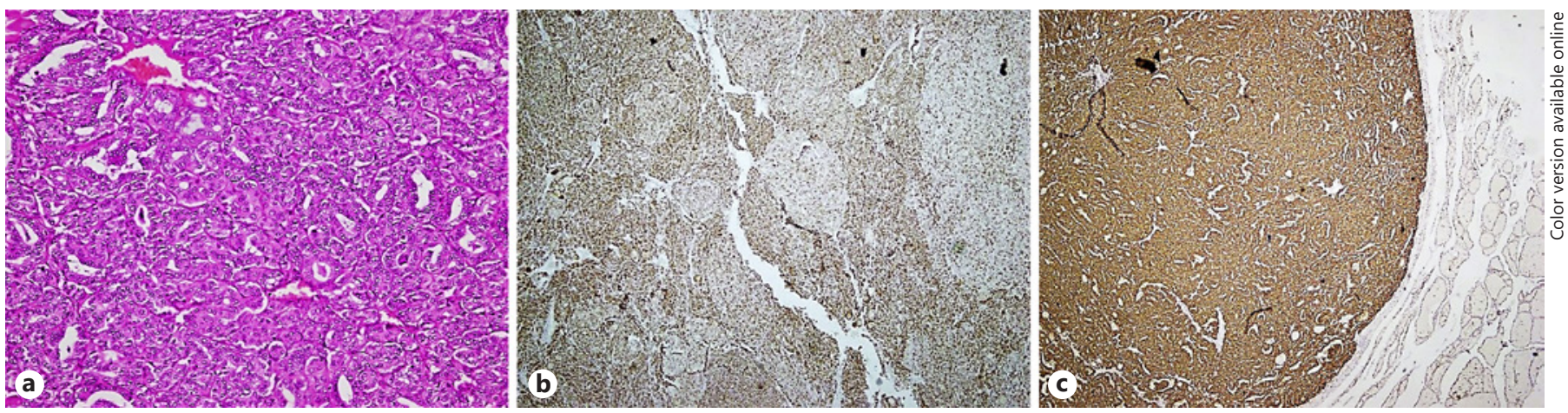

Fig. 4. a Follicular variant of papillary thyroid carcinoma, HE, $\times 400$. b Positive for HBME-1. c TROP-2 positivity.
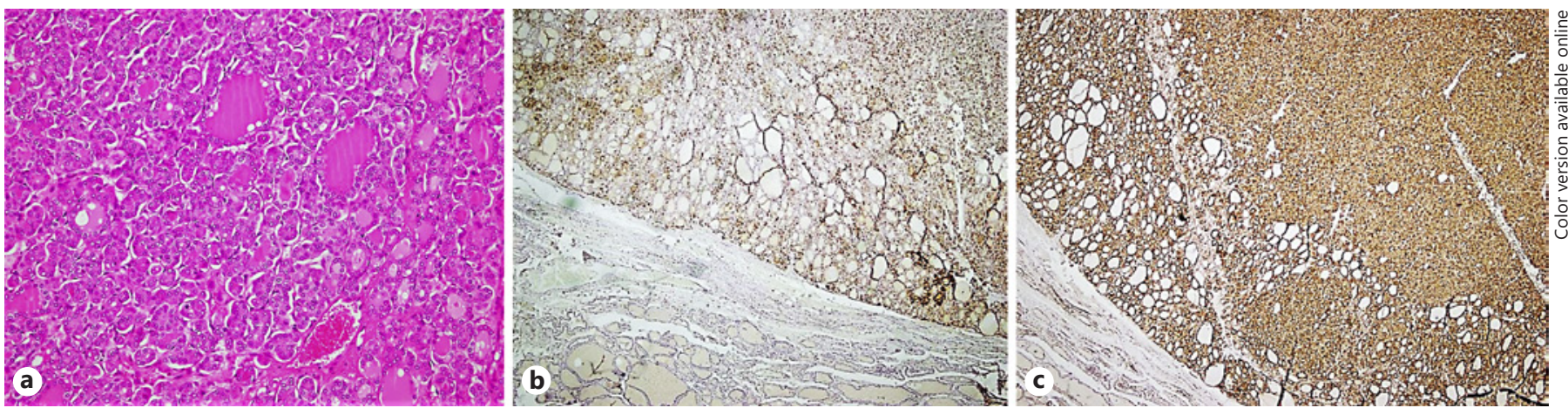

Fig. 5. a Noninvasive follicular neoplasm with nuclear features of papillary thyroid carcinoma, HE, $\times 250$. b Positive for HBME-1. c TROP-2 immunoreactivity.

Table 2. Sensitivity, specificity, PPV, and NPV of all benign versus malignant thyroid lesions for each IHC marker

\begin{tabular}{lllll}
\hline Marker & Sensitivity & Specificity & PPV & NPV \\
\hline HBME-1 & $84 \%$ & $98 \%$ & $98 \%$ & $83 \%$ \\
TROP-2 & $93 \%$ & $74 \%$ & $81 \%$ & $90 \%$ \\
\hline
\end{tabular}

PPV, positive predictive value; NPV, negative predictive value; IHC, immunohistochemistry.

(93.1\%) were positive for TROP-2. One of them (3.4\%) showed $2+, 1$ (3.4\%) showed $3+$, and 25 cases (86.2\%) showed $4+$ immunostaining (Fig. 3).

Of the 19 cases of FVPTC, 15 (79\%) showed immunostaining for HBME-1, and 18 (95\%) were positive for TROP-2. One case (4.8\%) showed $1+, 2$ cases (9.5\%) showed 2+, 9 cases (42.9\%) showed $3+$, and 8 cases (38.1\%) showed $4+$ immunostaining. Both cases of NIFTP were positive for HBME-1 and TROP-2 (Fig. 4, 5).
Table 2 shows the sensitivity, specificity, PPV, and NPV of TROP-2 and HBME-1 for differentiating malignant (PTC, FC, and FVPTC) from benign thyroid lesions (MNG, FA, and HCA). Both markers had a very good sensitivity (84\% for HBME-1 and $93 \%$ for TROP-2) and specificity (98\% for HBME-1 and 74\% for TRPO-2) in differentiating between benign and malignant thyroid lesions $(p<0.00001)$.

\section{Discussion}

The diagnosis of the majority of thyroid neoplasms is straightforward [13], but sometimes differentiating between benign and malignant lesions can be a difficult task, especially in the diagnosis of follicular patterned lesions, particularly encapsulated ones [1-4]. Ancillary studies, such as IHC markers, can be used to distinguish between equivocal benign and malignant thyroid lesions [4], but no single marker is sensitive nor specific enough to be used alone for this purpose. Thus, a combination of 
an IHC panel consisting of 2 or more markers may be required. In this study, we evaluated the utility of TROP-2 and HBME- 1 in the diagnosis of thyroid malignancies. In our study, HBME- 1 positivity was noted in $84 \%$ of malignant cases, while $97.8 \%$ of benign lesions were negative. Although focal weak reactivity was seen in few cases of FA, no MNGs were positive for this marker.

In the malignant group, the majority of PTCs $(27 / 29$, 93\%) and FVPTCs $(17 / 21,81 \%)$ and half of FCs $(3 / 6$, $50 \%$ ) showed strong and diffuse reactivity with HBME-1. Saleh et al. [4] showed that $87 \%$ of thyroid malignancies, $17.3 \%$ of nonneoplastic hyperplastic nodules, and $56.5 \%$ of benign neoplastic lesions were positive for HBME-1. Carcinomas presented strong and diffuse HBME-1 staining, but benign thyroid lesions (neoplastic and nonneoplastic) had focal and weaker staining. They concluded that HBME-1 was not a good IHC marker to differentiate between adenomas and carcinomas because half of the adenomas showed immunoreactivity with this marker [4].

Abd-El Raouf and Ibrahim [2] studied the IHC expression of HBME- 1 and galectin 3 in the differential diagnosis of follicular-derived thyroid nodules. They showed that both markers had diffuse reactivity in malignant lesions (FC, PTC, and FVPTC) and focal staining in benign neoplastic (FA) and benign nonneoplastic (MNG) lesions. The sensitivity and specificity of the HBME- 1 staining in malignant lesions were 89.3 and $66.7 \%$, respectively [2].

Palo and Biligi [12] proved that HBME-1 was the most sensitive and specific marker in distinguishing benign from malignant thyroid lesions [12]. Also, Mataraci et al. [7] demonstrated a higher rate of membranous (luminal) staining of HBME-1 in malignant thyroid lesions and a higher rate of cytoplasmic staining in benign lesions. In all of these studies, in concordance with our study, the percentage of positivity was higher in PTC and FVPTC than in FC.

In our study, 27/29 (93.1\%) of PTCs, 20/21 (95.2\%) of FVPTCs, and 5/6 (83.3\%) of FCs had strong membranous staining for TROP-2. The majority of carcinomas (82\%) demonstrated $3+$ or $4+$ staining. Three cases of MNGs $(13.6 \%)$ and 9 cases of FAs (37.5\%) were also positive for TROP-2. TROP-2 showed 93\% sensitivity, $74 \%$ specificity, $81 \% \mathrm{PPV}$, and $90 \% \mathrm{NPV}$ in differentiating between benign and malignant thyroid lesions. In our study, Hurthle cells and areas of Hashimoto's thyroiditis showed TROP-2 reactivity, so TROP-2 is not a good marker for malignancy in thyroid lesions with oncocytic change. Therefore, it is better to use another IHC marker (such as

TROP-2 and HBME-1 in Diagnosis of Thyroid Carcinoma
HBME-1) or molecular studies together with TROP-2 for differentiating between benign and malignant thyroid lesions with Hurthle cell morphology. None of the normal thyroid tissue or colloid particles stained with TROP-2. Liu et al. [6] evaluated TROP-2 expression in thyroid neoplasms. PTCs showed strong membranous staining with most staining $3+$ or $4+$, but most FCs as well as benign neoplasms were negative for this marker. In their study, normal thyroid tissue showed weak cytoplasmic (without membranous) staining. They stated that lining of degenerative cysts may show TROP-2 immunoreactivity as in 2 of 22 cases of MNGs in our study. They recommended that a small initial IHC panel including TROP-2 and HBME-1 was accurate for the classification of thyroid follicular patterned lesions with equivocal cytologic features of classic PTC [6]. Bychkov et al. [8] examined TROP-2 IHC as a highly accurate marker in the differential diagnosis of PTCs. They showed that nonneoplastic lesions, including nodular goiter, Hashimoto's thyroiditis, and Grave's disease, did not show TROP-2 staining. All FAs (including 12 oncocytic variants) and FCs were negative for TROP-2. They found that TROP-2 was a very sensitive $(98.1 \%)$ and specific (97.5\%) marker for PTCs. They recommended that TROP-2 could be useful for distinguishing between PTC and challenging nonneoplastic lesions, such as papillary hyperplasia in thyroid nodules; also, it can help to differentiate between PTC and its variants with solid components from FC. In contrast to other studies, we demonstrated that about $80 \%$ of our FC cases were positive for TROP-2. When we evaluated the immunoreactivity of HBME- 1 or TROP-2 in malignant thyroid lesions, the sensitivity and specificity both increased to $98 \%$ with a PPV of $98 \%$ and an NPV of $97 \%$. For interpreting HBME-1 expression, the pathologist should be aware of nonspecific colloid staining which may result in a false diagnosis, but this pitfall is not seen with TROP-2 staining.

In conclusion, we demonstrated that combination usage of TROP-2 and HBME-1 can reliably diagnose carcinoma with equivocal morphology with high sensitivity and specificity. These 2 markers specifically show higher immunoreactivity in PTCs and its variants than in FCs. Regarding the TROP-2 immunoreactivity, carcinomas show diffuse strong membranous immune reactivity in contrast to benign lesions, in which the reactivity was focal or weak. There is one exception to this finding; Hurthle cell neoplasms were strongly positive for TROP-2, so this marker may not be useful in the differential diagnosis of benign and malignant lesions with oncocytic morphology. 


\section{Acknowledgements}

The authors would like to thank Shiraz University of Medical Sciences, Shiraz, Iran, and also the Center for Development of Clinical Research of Nemazee Hospital, and Dr. Nasrin Shokrpour for editorial assistance.

\section{Statement of Ethics}

The study was approved by the ethics committee of Shiraz University of Medical Sciences.

\section{Disclosure Statement}

The authors have no conflicts of interest to disclose.

\section{Author Contributions}

Dr. Mokhtari contributed to the study design, pathology slide review, IHC slide review, and article writing. Dr. Zargari contributed to case selection, statistical analysis, and article writing.

\section{References}

1 Baloch ZW, Harrell RM, Brett EM, Randolph G, Garber JR; AACE Endocrine Surgery Scientific Committee and Thyroid Scientific Committee. Managing thyroid tumors diagnosed as non-invasive follicular tumor with papillary like nuclear features (NIFTP). Endocr Pract. 2017. doi: 10.4158/EP171940. DSC.

2 Abd-El Raouf SM, Ibrahim TR. Immunohistochemical expression of HBME-1 and galectin-3 in the differential diagnosis of follicularderived thyroid nodules. Pathol Res Pract. 2014 Dec;210(12):971-8.

3 Liu H, Lin F. Application of immunohistochemistry in thyroid pathology. Arch Pathol Lab Med. 2015 Jan;139(1):67-82.

4 Saleh HA, Jin B, Barnwell J, Alzohaili O. Utility of immunohistochemical markers in differentiating benign from malignant follicular-derived thyroid nodules. Diagn Pathol. 2010 Jan;5(9): 9
5 Paunovic I, Isic T, Havelka M, Tatic S, Cvejic D, Savin S. Combined immunohistochemistry for thyroid peroxidase, galectin-3, CK19 and HBME- 1 in differential diagnosis of thyroid tumors. APMIS. 2012 May;120(5):36879.

6 Liu H, Shi J, Lin F. The potential diagnostic utility of TROP-2 in thyroid neoplasms. Appl Immunohistochem Mol Morphol. 2017 Sep; 25(8):525-533.

7 Mataraci EA, Ozgüven BY, Kabukçuoglu F. Expression of cytokeratin 19, HBME-1 and galectin-3 in neoplastic and nonneoplastic thyroid lesions. Pol J Pathol. 2012 Mar;63(1): 58-64.

8 Bychkov A, Sampatanukul P, Shuangshoti S, Keelawat S. TROP-2 immunohistochemistry: a highly accurate method in the differential diagnosis of papillary thyroid carcinoma. $\mathrm{Pa}$ thology. 2016 Aug;48(5):425-33.

9 Muzafar A, Bukhari MH, Qureshi IU. A study of Galactin-3 on fine needle aspiration as a diagnostic marker differentiating benign from malignant thyroid neoplasm. Pak J Med Sci. 2017 May-Jun;33(3):726-31.
10 Arcolia V, Journe F, Renaud F, Leteurtre E, Gabius HJ, Remmelink M, et al. Combination of galectin-3, CK19 and HBME-1 immunostaining improves the diagnosis of thyroid cancer. Oncol Lett. 2017 Oct;14(4):4183-9.

11 Kong JS, Kim HJ, Kim MJ, Kim A, Lee D, Han $\mathrm{K}$, et al. The significance of TROP2 expression in predicting BRAF mutations in papillary thyroid carcinoma. J Pathol Transl Med. 2018 Jan;52(1):14-20.

12 Palo S, Biligi DS. Differential diagnostic significance of HBME-1, CK19 and S100 in various thyroid lesions. Malays J Pathol. 2017 Apr;39(1):55-67.

13 Bose D, Das RN, Chatterjee U, Banerjee U. Cytokeratin 19 immunoreactivity in the diagnosis of papillary thyroid carcinoma. Indian J Med Paediatr Oncol. 2012 Apr;33(2):107-11.

14 Guan H, Guo Z, Liang W, Li H, Wei G, Xu L, et al. Trop2 enhances invasion of thyroid cancer by inducing MMP2 through ERK and JNK pathways. BMC Cancer. 2017 Jul;17(1):486. 